(Göttingen), Sir G. Greenhill (London) and H. Fehr (Geneva) be continued in power and that, at its request, David Eugene Smith, of New York, be added to its number; that the delegates be requested to continue their good offices in securing the cooperation of their respective governments, and in carrying on the work; and that the Commission be requested to make such further report at the Sixth International Congress, and to hold such conferences in the meantime as the circumstances warrant.

VIRGIL SNYDER.

\title{
THE MÜNSTER MEETING OF THE DEUTSCHE MATHEMATIKER-VEREINIGUNG.
}

THE annual meeting of the Deutsche Mathematiker-Vereinigung was held in affiliation with the eighty-second convention of the association of German naturalists and physicians at Münster in Westphalia, September 15 to 19, under the presidency of Professor W. v. Dyck.

As usual, ample provision was made for the entertainment of the guests, a number of excursions being arranged for the afternoons, and a reception or concert each evening. Perhaps the most interesting excursion was that to the neighboring city of Essen, to inspect the works of the Krupp manufacturing company. The guests were shown the manifold processes of casting and hardening steel, the preparation of armor, the boring of cannon, etc., and were also furnished an opportunity of seeing the domestic and social problems and usages connected with this gigantic enterprise.

The session of Tuesday afternoon was devoted to the administrative affairs of the society. Reports of the status of the Encyclopedia and of the International commission on mathematical instruction were read, as well as a statement concerning the publication of various other works in which the society is interested, primarily those of Schroeder and of Euler. At this session Professor W. KILling read a paper "On the preparation of the gymnasium teacher," which was followed by a general discussion.

The session of Wednesday morning was held jointly with the section of physics, upon the invitation of the latter, to listen to the following more general reports in mathematical physics: 
(1) D. Hilbert (Göttingen): "Begründung der elementaren Strahlungstheorie."

(2) W. Nernst (Berlin): "Ueber den Energiegehalt der Gase."

(3) V. Smoluchowski (Lemberg): "Experimentell nachweisbare, der üblichen Thermodynamik widersprechende Molekularphänomene.”

Professor Smoluchowski presented a systematic resumé of certain phenomena, obtained experimentally, based upon a general formula of statistical mechanics derived from the fact that molecular systems execute small automatic oscillations from the state of thermodynamic equilibrium, according to the law of chance. Here belong in particular the Brownian molecular motion of particles suspended in liquids or gases, the distribution of emulsion particles in a gravitational field, the irregularity of the distribution of particles in solutions of collodion investigated by Svedberg, the appearance of opalescence in gases and mixtures in states approximating the critical ones, the blueness of the sky, certain manifestations of electric and optical phenomena observed in emulsions and in suspended particles, etc. The author called attention to certain other important phenomena, theoretically expected, which await experimental confirmation, and mentioned the width of the lines of the spectrum of Fabry, which, according to the Doppler principle, furnish an excellent justification of the Maxwell law of velocity. All these facts decide the long conflict between thermodynamics and kinetic theory in favor of the latter, so that it follows that the second fundamental law of thermodynamics, as formulated by Clausius, Thomson, and others is not true, since in small intervals of time and of space contradictory processes are continually going on, which are also observable in physical phenomena. The law is still applicable to unlimited fields of time and space.

Three sectional meetings were held to listen to papers presented to the society itself, the titles of which follow. On account of the Cambridge meeting of the Fifth international congress of mathematicians, the programme was rather shorter and the attendance slightly smaller than usual, but both were sufficient to make the Münster meeting an important one in the history of the society. 
It has been the custom during a number of years to pay particular attention to some one branch of mathematics at these meetings, and the proceedings have taken the form of symposiums and reports rather than the presentation of finished papers containing results of original research. While papers on other subjects were welcomed, the emphasis this year was primarily on differential geometry, and over half the papers treated of some phase of this subject.

(1) W. v. Drck (Munich): "Ueber die singulären Stellen der Differentialgleichungen erster Ordnung zweiten Grades."

(2) E. H. Moore (Chicago): "Remarks concerning relatively uniform sequences and series of functions."

(3) R. Rothe (Clausthal): " Anwendung der Vektoranalysis auf Differentialgeometrie."

(4) H. Wirner (Darmstadt): "Ueber eine geometrische Theorie der algebraischen Formen."

(5) F. Meyer (Königsberg): “Ueber einen verallgemeinerten Krümmungsbegriff."

(6) E. Salkowski (Charlottenburg): "Ueber die verschiedenen Begründungsarten der Differentialgeometrie."

(7) R. v. Lilienthal (Münster): " Ueber die Bestimmung der berührenden Kurve und Fläche bei Kurven- und Flächenscharen."

(8) W. Velten (Kreuznach): " Ueber die Funktionen, die aus der Jacobischen $\Omega$-Funktionen entspringen."

(9) A. Voigt (Frankfurt): "Mathematische Theorie des Tarifwesens."

(10) H. Mohrmann (Carlsruhe): "Ueber beständig hyperbolisch gekrümmte Kurvenstücke."

Abstracts of a number of the papers follow; the numbers correspond to those of the titles above.

3. Professor Rothe maintains that vector analysis in differential geometry is in fact of far greater importance than furnishing simply an abbreviation in the proofs and a convenient formulation of results. In the earlier investigations, originated by Grassmann, Hamilton, Möbius, the main question was the translation of formulas already known into the language of vectors; in this process many abridgements were found possible in the presentation and proofs. If we confine ourselves to vector analysis in the narrower sense (algebra) no simpler processes appear than those employed 
by Gauss, but when differential processes (analysis) are considered, it becomes a different matter. There we deal particularly with the gradient, divergence, and curl. By giving these concepts their proper physical meaning, and combining with them the usual elementary concepts of differential geometry, a great advance is possible. With every point in space a vector (usually one-valued) is associated, whose direction determines the lines of flow, and whose length determines the intensity of the field. Such considerations have been employed (among others) by Burali-Forti. He takes, for example, in the theory of surfaces as field vector the unit vector of the properly directed surface normal, and the lines of flow are determined as the lines of a normal congruence. This assumption materially simplified the formulas of the theory of surfaces.

After explaining the concept of a general field, a number of new results in the theory of surfaces were derived.

4. The theory of invariants of algebraic forms of order $\nu$ in one or more $\rho$-dimensional variables is contained within the theory of the forms which are linearly constructed from $\nu$ series $X, Y, \cdots, W$. The form $f(X, Y, \cdots, W)=a_{1} x_{1}+\cdots$ $+a_{\rho} x_{\rho}$ is a linear form of order $\nu$ in a space of $\rho$ (homogeneous) dimensions $R_{\rho}$ when its coefficients are linear forms of order $\nu-1$ in the series $Y, \cdots, W$. Equated to zero it defines a geometric correspondence, which associates $\nu-1$ arbitrarily chosen points $Y, \cdots, W$ as locus of the point $X$ with one element of $\rho-1$ dimensions (point, line, plane, ....) in space $R_{\rho}$. If by interchanging the series of $f$ new forms $f_{1}, f_{2}$, $\cdots f_{\tau-1}$ appear, then $\tau p=f+f_{1}+\cdots+f_{\tau-1}$ is a polar form, that is, in it any two rows may be interchanged. Moreover, the form $f$ is also associated with a null form $n=f-p$, that is, with a form which vanishes when the coordinates of any arbitrary point are substituted in all the series; it can always be written in such a manner that every term contains a factor $\left(x_{i} y_{k}-x_{k} y_{i}\right)$. The Clebsch-Gordan development in series divides a form $f$ into its polar form and null form, and further divides the latter into forms whose terms contain $1,2,3, \cdots$ factors of the designated kind, while the associated remaining factor is a polar form. At the Dresden meeting of the Vereinigung, Professor Wiener explained the process of transvection as applied to binary forms, and thus reduced the 
determination of the invariants of any form to the problem of determining the associated polar form of any given linear form. In the extension to forms in $\rho$ variables (so far as completed) he employs, as method of construction, only the geometric construction of the linear forms and their linear systems, and as means of proof the permutation of the series and the Galois groups generated by it. In this manner, the application of any symbol in the new theory that does not have a geometric meaning is rejected.

6. In the paper of Professor Salkowski, the various directions of propagation along a curve were considered and the various concepts of curvature systematized. In particular, the value and significance of various related ideas, like the synthetic methods of Schell and of Mannheim, were criticized. A more extensive summary will appear in an early number of the Jahresbericht.

7. Leibnitz and l'Hospital were familiar with the fact that the various curves of a one-parameter family are all touched by one curve. Monge extended the idea to surfaces (Applications, $\S 6$ ), and introduced the word envelope to define the surface thus generated, the word being at once suggested by visualization. If, for example, we take a cylinder whose cross-section extends to infinity on both sides of an inflexional tangent, and move the cylinder along the tangent, a family of surfaces results, each of which is touched by the same plane. Professor Lilienthal considered, in his Differentialgeometrie, Band I, the conditions under which the curves of a cross-section have as envelope the same section of the envelope of the surfaces. The same method is here immediately extended to surfaces.

Given a family of surfaces $f(x, y, z, v)=0$, and a family of space curves

$$
\begin{gathered}
f=0, \quad g(x, \quad y, \quad z, \quad v)=0 ; \\
x=f_{1}(u, v), \quad y=f_{2}(u, v), \quad z=f_{3}(u, v) ;
\end{gathered}
$$

the equations

$$
\frac{\partial f}{\partial v}=0, \quad \frac{\partial g}{\partial v}=0
$$


are satisfied by (1). Conversely, if (1) and (3) satisfy $x=\varphi_{1}(v), y=\varphi_{2}(v), z=\varphi_{3}(v)$, the curve defined by these equations is a contact curve, provided the determinants $f_{y} g_{z}-f_{z} g_{y}$, etc., do not all vanish for $x=\varphi_{1}(v)$, etc. If they do vanish, the equations must be further examined. For the surfaces defined by (2) we consider the determinants

$$
\frac{\partial f_{2}}{\partial u} \cdot \frac{\partial f_{3}}{\partial v}-\frac{\partial f_{2}}{\partial v} \cdot \frac{\partial f_{3}}{\partial u}
$$

etc. If they do not all vanish we have a contact curve $u=\varphi(v)$, provided $\partial f_{i} / \partial u(i=1,2,3)$ do not vanish. We now assume that the $u$ series and $v$ series are orthogonal; then $\partial x / \partial v$, etc., vanish along the contact curve. The series of contact curves lie on a surface. We consider the curve in the region of an ordinary point, at which not all the $u$-derivatives vanish, and the normal planes of the surface. Let the plane $N_{1}$ contain the tangent to the contact curve, and the plane $N_{2}$ be perpendicular to $N_{1}$ and cut the surface in the curve $L$, and let $\nu$ be the smallest value of $n$ for which the derivatives $\partial^{n} x / \partial v^{n}, \ldots$ do not all vanish. For the coordinates of the.point of intersection of $v+\Delta v=$ const. and $L$ we have expansions of the form

$$
x+\frac{1}{\nu !} \frac{\partial^{\nu} x}{\partial v^{\nu}}(\Delta v)^{\nu}+\cdots
$$

and

$$
(\xi-x) \frac{\partial^{v} x}{\partial v^{v}}=0
$$

is the equation of $N_{1}$. For odd values of $\nu$, the part of the curve $L$ corresponding to positive values of $\Delta v$ lies on one side of $N_{1}$, and that corresponding to negative values on the other. The contact curve is therefore not a proper envelope. For even values of $\nu$ two cases are possible; first, $\Delta v>0$ may correspond to a different part of the curve $L$ than $\Delta v<0$, in which case the curve $L$ has a cusp at its point of intersection with the contact curve, which is an edge of regression on the surface; if, however, both signs of $\Delta v$ correspond to the same point of $L$, from (4) no conclusions can be drawn. In this case the contact curve is an envelope of the series $v=$ const. This can be seen by rolling the tangents of a plane curve around a cylinder. 
Given the family of surfaces $f(x, y, z, v)=0$, and the curves

$$
f=0, \quad \partial f / \partial v=0
$$

(characteristics); if the equations

$$
f=0, \quad \partial f / \partial v=0, \quad \partial^{2} f / \partial v^{2}=0
$$

have a complete solution, corresponding geometrically to a curve, this curve is a contact curve of the series of characteristics, provided

$$
\frac{\partial f}{\partial y} \cdot \frac{\partial^{2} f}{\partial z \partial v}-\frac{\partial f}{\partial z} \frac{\partial^{2} f}{\partial y \partial v}, \text { etc., }
$$

are not all zero. It is not necessarily an envelope or an edge of regression. Monge's "arête de rebroussement" is not entirely justified, as is seen in the example of the circles of curvature and spheres of curvature of a space curve.

8. Dr. Velten's paper will appear in full in the next number of the Jahresbericht.

9. Professor Voigt first pointed out a number of serious errors in the current mathematical theory of taxes and tariff, and mentioned the remedy found in his recent book: Mathematische Theorie des Tarifwesens (Jena, 1912), namely, by means of a rational construction of the tariff from a mathematical basis. Certain advantages to both the importer and to the government were explained which would ensue from this procedure.

Virgil SNYder.

\section{SHORTER NOTICES.}

Differential and Integral Calculus. By Professor L. S. HuLBURT. New York, Longmans, Green, and Co., 1912. xviii $+481 \mathrm{pp}$. with figures.

THE subtitle of this volume, "An introductory course for colleges and engineering schools," indicates the scope of the author's aims. In view of the numerous elementary text books on the calculus, each enjoying more or less popularity at the present time, it might seem a priori that a newcomer in the field would have difficulty in displaying sufficient individuality to warrant its entrance upon the stage. But no 